\title{
THE EFFECT OF FOCAL LESIONS OF THE BRAIN UPON AUDITORY AND VISUAL RECENT MEMORY IN MAN
}

BY

\author{
LUCJAN STĘPIEÑ and STANISŁAW SIERPIŃSKI
}

\author{
From the Department of Neurosurgery, Polish Academy of Sciences, Warsaw, Poland
}

The problem of recent memory and its impairment caused by focal lesions of the brain has in recent years aroused an increasing interest among experimental and clinical neurophysiologists.

Earlier reports by Grünthal (1947), Glees and Griffith (1952), and Scoville (1954), as well as recent studies by Scoville and Milner (1957) and Penfield and Milner (1958), have shown the importance of the hippocampal complex for the normal processes of memory retention in man. We now know that bilateral destruction of these medial structures of the temporal lobe (the uncus, the amygdaloid nucleus, the hippocampus, and the hippocampal gyrus) may cause severe and persistent disturbance of recent memory while leaving essentially intact other aspects of human behaviour such as old stable memories, the performance of professional skills, and so-called general intelligence. Even unilateral temporal lobectomy may be responsible for an immediate and grave loss of recent memory-as is usually the case after bilateral lesions-when the electroencephalographic examination points to independent abnormality in both inferior temporal regions.

It is regrettable that there should be so few animal experiments bearing on this general topic. It is a fact that, so far, psychophysiology has been concerned almost exclusively with the investigations of old or static memory as opposed to recent or dynamic memory. One of the reasons for this paucity of information in animal experiments can probably be found in the lack of appropriate tests which will give a true measure of this phenomenon. As pointed out recently by Konorski (1959), those tests used in the past, such as the delayed response tests, conditioned inhibition, or trace conditioned reflexes, are open to criticism with regard to their potentiality as true indicators of recent memory performances in respect of the modalities of stimuli used in these tests. For this reason he suggests the use of a new testing procedure which eliminates most of the criticisms. This test uses compound stimuli and each stimulus is made up of two signals of the same sensory modality separated by a short interval which may be varied from one to five seconds or more. As used in Konorski's laboratory a compound stimulus consisting of two identical signals, e.g., two tones of identical pitch, is positive and is reinforced by food if it produces the appropriate response in the animal. On the other hand, when the second signal of a compound stimulus differs from the first, e.g., two tones of different pitch, the stimulus is negative and is not reinforced. In other words the compounds Sx-Sx and Sy-Sy . . . etc. are positive while Sx-Sy and Sy-Sx are negative a long as $x$ differs from $y$.

The interesting feature in this testing procedure and what distinguishes it from previously used tests is that here the first signal has no significance io itself; it can be part of either a positive or a negativg compound stimulus. The correct response is possibe only when the animal retains the trace of the first. signal up to and including the time of the second $\overrightarrow{0}$ signal, so that the two can be compared. Thus when the first component of the compound stimulus is applied the animal does not "know" whether he will get reinforcement or not, because this depends on the comparison of the second component with the first one. Consequently, the animal cannot anticipate a particular kind of reaction, as is the case in delayed responses or in other tests in which the first signal of the compound already determines the positive or negative character of the conditioned reaction.

There is some evidence in support of the view that recent memory traces of a given sensory modality may be irreversibly destroyed following lesions lying outside the sensory cortical projection areas.

Chorążyna and Stẹpien (1960) applied the test described above for recent memory in their studies on auditory recent memory in dogs. After the animals had been trained to differentiate between the pairs of any identical tones (Sx-Sx) and any different tones (Sx-Sy), the area situated ventrally to the auditory projective area, namely, the gyrus 
Sylviacus anterior and posterior, was removed bilaterally. After this operation the dogs lost completely and permanently the ability to differentiate this test, although not only simple differentiation but even conditioned inhibition was fully preserved. On the other hand, bilateral ablation of the prefrontal area, gyrus proreus and orbitalis, or of the projective auditory area, gyrus ectosylvius, did not impair the performance of our test (Chorążyna and Stępień, 1960).

Similar results were obtained by Goldberg, Diamond, and Neff (1957) in cats. After bilateral ablation of the "insular and temporal cortex" ventral to the second auditory area AII, the discrimination between groups of tones which differ only in temporal pattern was lost, although simple tone discriminations were preserved.

Even more convincing results were recently obtained by Stępień, Cordeau, and Rasmussen (1960) in their studies on auditory and visual recent memory in monkeys. After the animals had been trained to differentiate between the pairs of identical and different rhythmic stimuli, both acoustic (clicks) and visual (flashes), different parts of the temporal lobe were removed bilaterally. After ablation of anterior parts of the first and second temporal gyri differentiation between the pairs of auditory stimuli was lost, while the performance of the same test with visual stimuli was preserved. The opposite was found after the ablation of posterior parts of the second and third temporal gyri and the gyrus fusiformis. After bilateral removal of the hippocampal complex (the uncus, amygdaloid nucleus, hippocampus, and hippocampal gyrus) differentiation of both auditory and visual tests was abolished.

To sum up, there is increasing evidence that "the recording of experience can be localized in the same sense that sensory functions and speech functions can be. Obviously, none of these subdivisions is separable from the work of the brain as a whole" (Penfield and Milner, 1958). We believe that the cortical areas lying in the immediate neighbouring of the sensory cortical projection areas as well as the hippocampal region are of critical importance for the maintenance of recent memory traces.

In our department we are studying auditory and visual recent memory in patients with focal lesions of the brain, and the purpose of this report is to describe the method and the results of our investigations, as well as to present one interesting case of temporal lobe epilepsy with recent memory impairment which cleared completely after removal of an epileptogenic area in the right fronto-temporal region of the brain.

\section{Method of Examination of Recent Memory}

All examinations of recent memory have been performed using the method described by Konorski (1959) with all precautions indispensable in neurophysiological experiments. We applied the compound auditory and visual stimuli, the duration of the presentation period for each signal being two seconds and the interval between two signals of the compound from 10 to 120 seconds. The pairs of identical and different pitch of tones or the red light of identical and different intensity of brightness were used in our examinations. The stimulus tones and lights were produced by a generator apparatus with an automatic timer which controlled the duration of the stimulus and registered the strength and the latent period of reaction. As the aim of our investigations was the examination of recent memory and not of the ability to perform difficult or delicate differentiations of the auditory and visual stimuli, we always applied very large differences in tones as well as in brightness of the red light.

At the beginning of the examination we explained the apparatus to the patient and he was instructed to press the spring connected with the recording instrument when the two signals of the compound auditory or visual stimulus would be identical. On the other hand, when the second signal would differ from the first, he should leave the spring alone. These instructions were repeated and trials given until we were quite sure that the patient understood the task.

When the patient showed no difficulty in differentiation with an interval of 120 seconds between two signals, the task was made more difficult by introducing in the middle of the interval an additional, distracting stimulus, which turned the patient's attention from the proper task, i.e., from keeping in mind the recent memory traces of the first signal. The distracting stimulus was always from another analyser than the one by which recent memory was examined. For example, during examination of auditory recent memory the distracting stimulus, given in the interval between two tones, was the red or green light. When the red light was applied the patient should press the spring of the recording apparatus but after lighting the green lamp he should leave the spring alone.

The daily test period usually consisted of 10 to 30 trials, depending on the length of the intervals and on the general health of the patient. On the first sign of weariness we stopped the examination.

Usually, we performed recent memory examinations before as well as after operation. We were able, therefore, to compare the effect on recent memory produced by increased intracranial pressure and by focal lesions of the brain alone.

\section{Results}

Control Group.-As a control group we examined 12 patients with peripheral nerve injuries or genuine epilepsy. No difficulties were observed in differentiation in our test for recent memory with 120 seconds' interval between two signals and with a distracting stimulus in the middle of this interval. 
Patients with Focal Lesions of Brain.-In this group 50 patients with focal lesions of the brain were examined: gliomas 37 , meningiomas 7 , traumatic lesions 3 , abscess 2 , and sclerotic abnormality in the hippocampus 1 . In all cases the character of the lesion, its localization and extent could be precisely determined by operation and microscopic examination.

In 15 cases the lesion was situated in the frontal lobe (12 in the left and three in the right hemisphere); in 15 cases in the parietal lobe (seven in the left and eight in the right hemisphere); in 15 in the temporal lobe (11 in the left and four in the right hemisphere); in four the junction of the left temporal, parietal, and occipital lobe was damaged; in one case of malignant glioma the whole left hemisphere of the brain was removed; and in the last one, sclerosis of the right amygdalo-hippocampal complex was found (Table I).

TABLE I

LOCALIZATION OF FOCAL BRAIN LESIONS IN 50 CASES

\begin{tabular}{l|c|c|c}
\hline & $\begin{array}{c}\text { Left } \\
\text { Hemisphere }\end{array}$ & $\begin{array}{c}\text { Right } \\
\text { Hemisphere }\end{array}$ & Total \\
${ } }$ & 12 & 3 & 15 \\
Temporal lobe & 11 & 4 & 15 \\
Parietal lobe \\
Temporo-parietal- \\
$\begin{array}{l}\text { occipital junction } \\
\text { Hemispherectomy }\end{array}$ & 4 & 8 & 15 \\
\hline Total & 1 & - & 4 \\
\hline
\end{tabular}

On the basis of our present investigations we may assume that unilateral lesions of the frontal or parietal and/or temporal lobe, either right or left (dominant) hemisphere cause no impairment of auditory or visual recent memory. Even in those cases in which the intracranial pressure before operation was greatly increased, the differentiation in the recent memory test was normal.

Impairment of recent memory performance was found in only three patients: (1) after nearly complete left hemispherectomy; (2) in a patient with a malignant glioma of the left temporal lobe infiltrating the insula and optic thalamus; and (3) in an epileptic patient with sclerotic abnormalities in the amygadalo-hippocampal region.

Case 1.-C.B., aged 34 years, was operated upon three times because of a malignant glioma of the left hemisphere of the brain. During the third operation performed on April 2, 1958, almost the whole left hemisphere was removed. The tumour infiltrated the nucleus caudatus and the optic thalamus.

Neurological examination revealed right hemiplegia and hemianaesthesia, a right homonymous hemianopsia, severe disturbances in verbal repetition, understanding, and nomination. The patient was apathetic and poorly orientated in time and place.
Recent memory examinations were performed four weeks after operation. The patient was able to differentiate the auditory test only when the interval between two tones was not longer than five seconds. The visual task was solved with an interval between two signals as long as 30 to 60 seconds (Table II).

TABLE II

RESULTS OF RECENT MEMORY EXAMINATIONS IN C.B.

\begin{tabular}{c|c|c|c|c}
\hline \multirow{2}{*}{$\begin{array}{c}\text { Interval between } \\
\text { Two Signals } \\
\text { (sec.) }\end{array}$} & \multicolumn{2}{|c|}{ Auditory Test } & \multicolumn{2}{c|}{ Visual Test } \\
\cline { 2 - 3 } & Trials & Errors & Trials & Errors \\
\hline 2 & 20 & 1 & - & - \\
5 & 37 & 2 & 10 & 0 \\
10 & 60 & 19 & 10 & 0 \\
20 & 20 & 7 & 10 & 0 \\
30 & 10 & 4 & 10 & 1 \\
60 & - & - & 12 & 1 \\
\hline
\end{tabular}

Case 2.-A.J., aged 50 years, was operated on on December 2, 1957, for a malignant glioma of the left temporal lobe infiltrating the insula and basal ganglia. The whole temporal lobe, the inferior part of the parietal, and the anterior part of the occipital lobe were removed. There was right hemiplegia and hemianaesthesia, and very grave disturbances of speech and disorientation for time and place.

Examination of recent memory four months after operation showed that the patient was completely unable. to differentiate between the auditory and visual test (Table III).

TABLE III

RESULTS OF RECENT MEMORY EXAMINATIONS IN A.J.

\begin{tabular}{c|c|c|c|c}
\hline \multirow{2}{*}{$\begin{array}{c}\text { Interval between } \\
\text { Two Signals } \\
\text { (sec.) }\end{array}$} & \multicolumn{2}{|c|}{ Auditory Test } & \multicolumn{2}{|c|}{ Visual Test } \\
\cline { 2 - 5 } & Trials & Errors & Trials & Errors \\
\hline 1 & 20 & 1 & 80 & 30 \\
5 & 158 & 46 & 51 & 15 \\
10 & 64 & 28 & - & - \\
\hline
\end{tabular}

In the first two cases recent memory disturbances were caused by large and deeply situated malignant tumours of the brain and were associated with other evidence of severe and widespread impairment of intellectual abilities, therefore they will not be the subject of our further analysis and discussion. On the other hand, the third case, in which recent memory deficit produced by a lesion in the hippocampal zone cleared completely after resection of an epileptogenic region, seems to be very interesting and will be presented in detail.

Case 3.-H.K., aged 15 years, was admitted to the Department of Neurosurgery, Polish Academy of Sciences, Warsaw, on September 11, 1959, with a history of recurrent seizures since the age of 6 months. These had been increasing in frequency and severity despite treatment by various anticonvulsant drugs. Her birth was thought to have been normal but she had had a convulsive seizure at the age of 6 months and the left hemiparesis was noted then. 
Seizure Pattern.-The attacks began with a sensation in the heart region, at times accompanied by palpitation and nausea. After this the eyes and head turned to the left, the left extremities became rigid and extended, followed by jerking movements in the arms and legs, beginning in the left arm. Afterwards there was a deeper hemiparesis of the left extremities for about 20 to 30 minutes. These attacks lasted for about two to five minutes and appeared usually every three or four days, but sometimes were repeated many times a day.

Physical Examination.-There was slight paresis of the lower portion of the left facial nerve and of both left extremities; the tendon and periosteal reflexes were all hyperactive on the left side. Examination of the blood and urine revealed nothing abnormal. The Wassermann test on blood and cerebrospinal fluid was normal.

Radiological Findings.-The plain films of the skull showed slight elevation of the floors of the anterior and middle fossae on the right. The pneumoencephalograms showed the ventricles to be of normal size, but all parts of the right lateral ventricle were very slightly wider than the corresponding parts on the left.

Electroencephalographic Findings.-Three electroencephalographic examinations were summarized by Dr. T. Bacia as follows:

"The electroencephalograms revealed continuous multiple spikes, multiple spike-and-wave patterns, and slow wave complexes at frequencies between 2 and 3 per second from the right frontal and temporal regions and phase reversals in the anterior part of the right temporal lobe. Sporadic sharp waves and wave-andspike complexes occurred in the left temporal region, $1 / 5$ second later in comparison with the abnormalities in the right temporal lobe."

Pre-operative Examination of Recent Memory.-The patient was a rather anxious young girl of average intelligence who showed no impairment of memory for the distant past or loss of attention, concentration, reasoning ability, or verbal recall. She was able to repeat nonsense syllables or series of numbers after even as long as five minutes.

The ability to discriminate our test for auditory and

TABLE IV

RESULTS OF RECENT MEMORY EXAMINATIONS IN H.K.

\begin{tabular}{c|c|c|c|c}
\hline $\begin{array}{c}\text { Interval } \\
\text { between } \\
\text { Two Signals } \\
\text { (sec.) }\end{array}$ & \multicolumn{2}{|c|}{$\begin{array}{c}\text { No Distraction } \\
\text { in Interval }\end{array}$} & \multicolumn{2}{c}{$\begin{array}{c}\text { Distraction } \\
\text { in Interval }\end{array}$} \\
\cline { 2 - 5 } & Trials & Errors & Trials & Errors \\
\hline $\begin{array}{c}\text { Auditory Recent } \\
\text { Memory: }\end{array}$ & & & & \\
10 & 12 & 1 & - & - \\
20 & 7 & 0 & - & $=$ \\
30 & 10 & 0 & $\overline{7}$ & $\overline{13}$ \\
60 & 10 & 0 & 10 & 6 \\
120 & 10 & 4 & 10 & \\
$\begin{array}{c}\text { Visual Recent } \\
\text { Memory: }\end{array}$ & 10 & & & - \\
20 & 10 & 0 & - & - \\
60 & 29 & 1 & 28 & 14 \\
\hline
\end{tabular}

visual recent memory revealed definite disturbances. She was able to perform the task only when the interval between two signals was 60 seconds and when she was permitted to keep her attention upon it. But when the distracting stimulus was interposed between the two signals of the compound stimulus she was quite unable to keep in mind the first signal and therefore the differentiation of both auditory and visual stimuli was destroyed (Table IV).

Operation.-On October 6, 1959, a right frontotemporal osteoplastic craniotomy and a partial frontal and temporal lobectomy were performed.

Under local anaesthesia, a curved incision was made and a fronto-temporal scalp and bone flap turned down. The dura was thick and dense adhesions were seen between the dura and leptomenings in the temporal region. When the dura was opened the sulci and blood vessels on the surface of the brain appeared more or less normal but there was some pallor of the cortex in the region of the operculum frontale and of the first and second temporal gyri. Electrocorticography showed abnormal discharges recorded by the electrodes which had been placed at the points marked in Fig. 1.

Electrical stimulation (50 cycles, 1 millisecond, 3-8 volts) outlined the fissure of Rolando and the stimulation of this region caused after discharges in the cortex of the temporal lobe (Fig. 1).

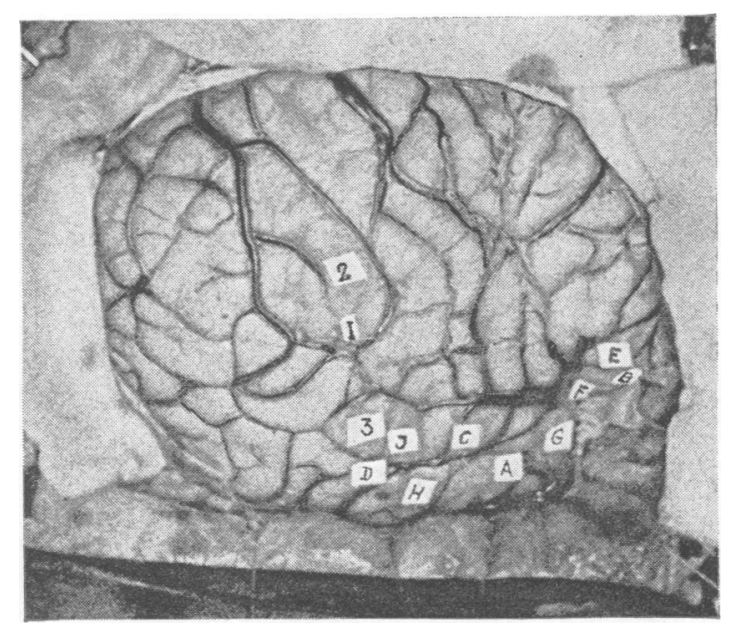

FIG. 1.-Operation photograph. Numbered tickets indicate sites of positive response to stimulation, lettered tickets sites of electrographic abnormality.

A-spikes, spike-and-wave complexes, and phase reversals B-slow waves

C-spikes

D, E-slow waves and spikes

F-spikes and phase reversals

G, $\mathbf{H}$-sharp waves

Point $1 / 3 \mathrm{v} /$ - Flexion of the index finger.

$2 / 3 \mathrm{v} /$-Nothing.

$2 / 4 \mathrm{v} /$-Flexion of the middle finger and the hand.

$3 / 3 \mathrm{v} /$-Nothing.

3/5v/-After discharges in points A, C, D, and $H$. 


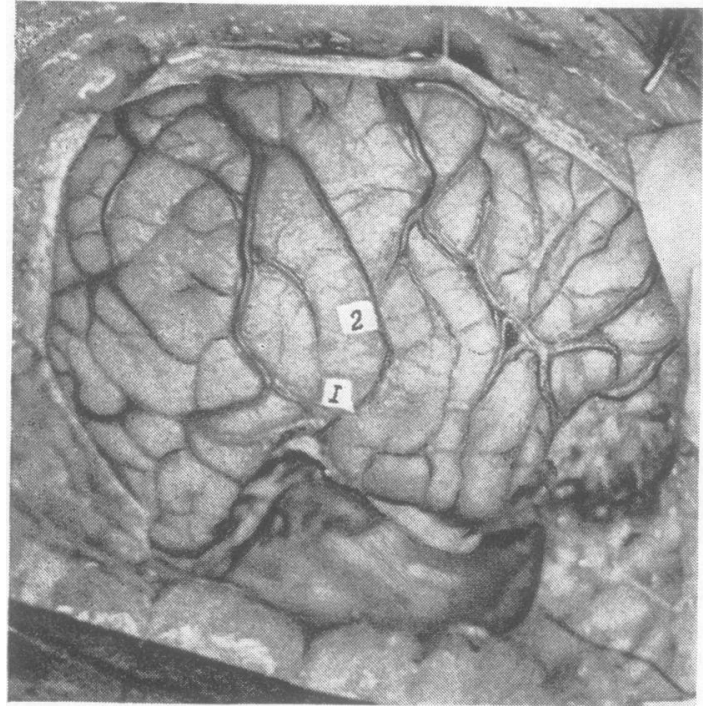

FIG. 2.-Operation photograph. Same as in Fig. 1, but after removal of epileptogenic areas.

It was concluded that there must be an epileptogenic zone of the cortex in the anterior part of the temporal lobe and in the operculum frontale and we decided to remove those areas. During the process of removal marked abnormality was encountered in the region of the amygdaloid nucleus and the hippocampus. It was typical of epileptogenic tissue, the gray matter being tough and yellow.

After removal of the anterior temporal lobe, including the whole of the uncus and hippocampal gyrus and the operculum frontale, a second electrocorticogram was carried out, which showed abnormal potentials of the epileptogenic variety still present in the lateral and inferior temporal frontier. Further brain tissue was removed and the electrocorticogram repeated. After this excision all high-voltage activity had disappeared.

The final line of removal is shown in the operation photograph (Fig. 2) and in the brain chart (Fig. 3). On the lateral surface of the temporal lobe the line of ablation was $6 \frac{1}{2} \mathrm{~cm}$. along the fissure of Sylvius and $7 \mathrm{~cm}$. along the inferior temporal surface. In the opercular region the removed cortex was triangular in shape with equal arms; the base of the triangle was $2 \frac{1}{2} \mathrm{~cm}$. along the fissure of Sylvius and the height $3 \mathrm{~cm}$.

Post-operative Course.-The post-operative course was uneventful. The neurological examination revealed a left upper quadrant homonymous visual field defect, but otherwise the status was as before operation. Unfortunately, she had one small attack on the second post-operative day.

Post-operative electroencephalograms showed residual abnormality over the right frontal and temporal regions but no epileptogenic focus was found (Dr. T. Bacia).

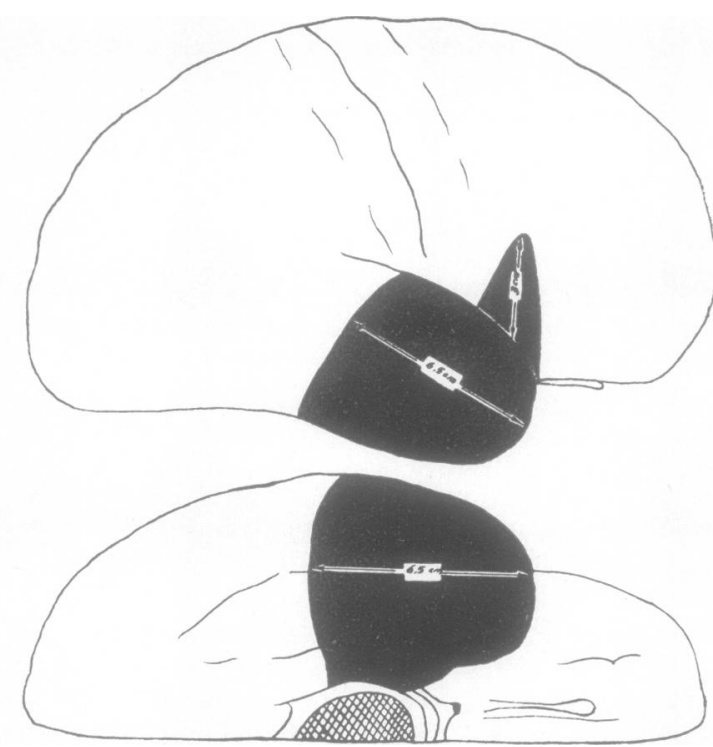

FIG. 3.-Diagram of the right hemisphere of the brain showing area of excision of temporal and fronto-opercular cortex.

Test of Recent Memory.-The most interesting feature of the post-operative course was the disappearance of ${ }^{\mathbb{D}}$ 음 all disturbances of recent memory, which were so clearly $\vec{\Phi}=$ seen before the operation. The examination of auditory $\frac{2}{\sigma} Z$ and visual recent memory showed that the patient had no difficulty whatever in differentiation in our test for recent memory even when the interval between twa signals was 120 seconds and when the distracting stimulus was given in this interval (Table V).

TABLE V

RESULTS OF RECENT MEMORY EXAMINATIONS IN H.K AFTER REMOVAL OF EPILEPTOGENIC ZONES

\begin{tabular}{c|c|c|c|c}
\hline $\begin{array}{c}\text { Interval } \\
\text { between } \\
\text { Two Signals } \\
\text { (sec.) }\end{array}$ & \multicolumn{2}{|c|}{$\begin{array}{c}\text { No Distraction } \\
\text { in Interval }\end{array}$} & \multicolumn{2}{|c}{$\begin{array}{c}\text { Distraction } \\
\text { in Interval }\end{array}$} \\
\cline { 2 - 4 } & Trials & Errors & Trials & Errors \\
\hline $\begin{array}{c}\text { Auditory Recent } \\
\text { Memory: } \\
60\end{array}$ & & & & \\
$\begin{array}{c}120 \\
\text { Visual Recent }\end{array}$ & 27 & 0 & 20 & 0 \\
Memory: & 20 & 0 & 21 & 0 \\
60 & & & & \\
120 & 20 & 0 & 20 & 0 \\
\hline
\end{tabular}

Thus we may assume that after the removal of an epileptogenic focus in the right temporal and frontal gyri which generated abnormal discharges in both temporal lobes, the patient had no more difficulty in differentiation in our tests for recent memory. This was also true in a control examination performed on January 22, 1960, three months after operation. 


\section{Discussion}

It is now almost generally accepted that there are two different physiological mechanisms underlying two different kinds of memory traces, e.g., of lasting effects produced in the central nervous system by stimuli impinging upon it. Stable or static memory is due to certain micromorphological changes, growing and multiplication of synaptic contacts between the axons (Ramon y Cajal, 1911; Child, 1921; Coghill, 1929; Konorski, 1948). The other kind-recent or dynamic memory-lasting only for a number of seconds, minutes, or hours, is attributed to the reverberating chains of neurons, activation of which may persist for a relatively long time after the actual stimulation of neurons (Hebb, 1949).

If we hold the view that the impulses which circulate in the reverberating circuits constitute the physiological basis responsible for recent memory, then the important problem arises of where these reverberating chains of neurons are situated. Since the classical experiments of Jacobsen (1936), who demonstrated that after bilateral ablations of the prefrontal area in monkeys the delayed responses are abolished, it was generally held that the normal function of frontal lobes of the brain is an indispensable condition for recent memory processes. However, the more accurate analysis of the impairment of the delayed responses after frontal lobe ablations, performed by Konorski and Lawicka (1959), showed that prefrontal lesions produce the deficit in recent memory of directional cues only instead of recent memory in general, as was stated by Jacobsen. We have some evidence to believe that after prefrontal ablations other forms of recent memory, e.g., auditory recent memory, are quite normal (Chorążyna and Stępień, 1960).

There is a great body of evidence showing that the associative areas of the temporal lobe play an important role in the physiological processes of recent memory. We know that bilateral ablations of the so-called associative auditory area (the area situated ventrally or anteriorly to the auditory projective area) produce severe and irreversible impairment of auditory recent memory but lesions in the associative visual area (the infero-posterior parts of the temporal lobe) cause deficit in visual recent memory, leaving other kinds of memory and the whole personality of the animal quite intact.

On the other hand, clinical and experimental data show that bilateral hippocampal lesions produce a striking deficit of recent memory in general.

Of 50 patients with focal unilateral lesions of the brain there were 15 cases with lesions in the temporal lobe (11 left and four right hemisphere) and four cases with lesions in the left temporo-parietal occipital junction. The lesions were so large that they should have covered the regions similar to associative areas (auditory and visual) in animals.

The results of our examinations seem to show that unilateral lesions, situated in the temporal, parietal or frontal lobe-independent of the side (dominant or subordinate hemisphere)-produce no impairment of auditory or visual recent memory. They are similar to those obtained in animal experiments and show that recent memory processes in man, as in animals, are connected with both cerebral hemispheres. The same results were obtained by Penfield and Milner (1958) in their study of recent memory in patients with temporal lobectomies. They performed the examinations of recent memory using psychological tests which showed general impairment of recent memory without giving the opportunity of more accurate analysis of different modalities of memory. Of over 90 patients with unilateral temporal lesions in the left or right hemisphere, loss of recent memory appeared only in two cases. In both cases the operation was in the dominant (left) hemisphere and the E.E.G. examinations before operation revealed two independent epileptogenic zones lying in both temporal lobes.

Our patient H.K. is a good example, showing the important role of the infero-ventral parts of the temporal lobes for recent memory and emphasizing that only bilateral lesions of these areas produce memory deficit. Before operation, when the epileptogenic zone in the right fronto-temporal region generated afterdischarges in the left temporal area, there were severe disturbances in differentiation of the auditory and visual test for recent memory. It was shown that the control group and the patients with unilateral temporal lesions had no difficulty in performing this task, even with an interval of 120 seconds between two signals and with the distracting stimulus applied in the middle of the interval. On the other hand, H.K. was quite unable to differentiate our test with a 60 seconds' interval between two signals if the distraction was introduced in the middle of this interval. It looks as if a distracting stimulus applied in the interval "washed out" the memory traces of the first signal and the patient, therefore, could not compare it with the second signal.

Very similar disturbances were found in patients described by Penfield and Milner (1958).

"They could retain in mind a sentence or a short sequence of numbers, provided they were permitted to keep their attention upon it, even as long as 15 minutes if no one spoke to them. But if they turned their attention to something else, even momentarily, they might forget the previous matter completely-might even forget that there was a previous matter."

Careful analysis of the electroencephalographic 
studies in H.K. revealed the epileptogenic zone in the right fronto-temporal region which generated afterdischarges in the left temporal lobe. According to Penfield's theory, we believe that the right hippocampal complex was completely destroyed and all memory processes must have been carried by the left temporal lobe, the function of which was also more or less completely impaired by the pathological afterdischarges. This was the reason for the recent memory deficit. After all epileptogenic areas lying in the right fronto-temporal region had been removed, the E.E.G. studies showed no pathological afterdischarges in the brain activity and at the same time the patient had no more difficulty in differentiation in our test for recent memory.

This conclusion seems to be in full agreement with Penfield's opinion that the hippocampal complex plays an important role in preserving the recent memory traces when the person turns his attention to something else. After bilateral ablation of this area an essential mechanism for recording and preserving conscious experiences is destroyed and therefore all recent memory traces are "washed out" by any new stimulus which turns the attention from previous experience. We do not know whether the hippocampal complex is the place where recent memory traces are laid down, or it may be that the hippocampal zone is only a half-way station leading the impulses to some other structures in the "centrencephalic system", which has duplicate connexions with the two hippocampal areas (Penfield and Milner, 1958).

Also it may be that a normal function of the hippocampal zone is that of an indispensable factor for the maintenance and consolidation of recent memory traces in some other cortical and subcortical areas of the brain. Numerous afferent and efferent connexions between hippocampus and thalamus and hypothalamus and centrencephalic system appear to fit the hippocampus for the role of a "universal analyser" of non-specific stimuli (Yakovlev, 1952) or of a "non-specific" or "catalytic" activator (Herrick, 1933; Grünthal, 1947), which is basic for the proper functioning of all specific cortical analysers and for higher integration of impulses.

\section{Summary}

Auditory and visual recent memory was examined in 50 patients with unilateral focal lesions of the brain using a new test for recent memory proposed by Konorski (1959).

Unilateral lesions situated in the temporal, parietal, or frontal lobe, quite independent of the side (dominant and subordinate hemisphere), cause no impairment of auditory or visual recent memory.

An interesting case is presented in which a deficit for recent memory produced by a lesion in the right hippocampal zone giving afterdischarges in both temporal lobes cleared completely after the radical removal of an epileptogenic focus in the right fronto-temporal region.

This is a confirmation of Penfield's theory that the hippocampal complex plays an important role in the normal processes of memory retention in man.

\section{REFERENCES}

Cajal, S. Ramon y (1911). Histologie du système nerveux de l'homm et des vertébrés. Maloine, Paris.

Child, C. M. (1921). The Origin and Development of the Nervout System. University of Chicago Press.

Chorążyna, H., and Stępien, L. (1960). The Effect of Bilaterd Removal of the Associative Auditory Area upon Auditory Recent Memory in Dogs. To be published.

Coghill, G. E. (1929). Anatomy and the Problem of Behaviour. Cambridge University Press, London.

Glees, P., and Griffith, H. B. (1952). Mschr. Psychiat. Neurol., 123 193.

Goldberg, J. M., Diamond, I. T., and Neff, W. D. (1957). Fed. (D. Proc., 16, 47 (abstr. No. 204).

Grünthal, E. (1947). Mschr. Psychiat. Neurol., 113, 1.

Hebb, D. O. (1949). The Organization of Behaviour. A Neuropsychological Theory. Wiley, New York.

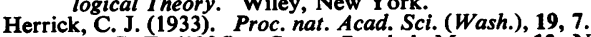

Herrick, C. J. (1933), Proc. nat. Acad. Sci. (Wash.), 19, 7. . 3 (Serial Jacobsen, C. F

Konorski, J. (1948). Conditioned Reflexes and Neuron Organization. trans. S. Garry. Cambridge, University Press, London. (1959). Bull. Acad. pol. Sci., Cl. VI, 7, 115.

- and Eawicka, W. (1959). Acta Biol. exp. (Lodz), 19, 175.

Penfield, W., and Milner, B. (1958). A.M.A. Arch. Neurol. Psychiat. 79,475 .

Scoville, W. B. (1954). J. Neurosurg., 11, 64.

Scoville, W. B. (1954). J. Neurosurg., 11, 64.

Stępień, L., Cordeau, P., and Rasmussen, T. (1960). The Effect of Temporal Lobe and Hippocampal Lesions on Auditory and Visual Recent Memory in Monkeys. To be published.

Yakovlev, P. I. (1952). Epilepsia (Boston), 3 ser., 1, 51. 